UDK 821.131.1-32.09Boccaccio G. UDK 821.112.2-223.09Sachs H.

\title{
EIFERSUCHT UND FRAUENLIST. BOCCACCIOS DECAMERON UND SEINE REZEPTION IN DER FRÜHEN NEUZEIT AM BEISPIEL VON HANS SACHSENS FASTNACHTSPIEL DER GROSS EYFERER, DER SEIN WEIB BEICHT HÖRET
}

\author{
Marija Javor Briški
}

Giovanni Boccaccio (1313-1375)

zum 700. Geburtstag

\begin{abstract}
Auf der Grundlage des sog. Zainer- (um 1476) und des Cammerlander-Druckes (1535) wird die produktive Rezeption der fünften Novelle des siebten Tages von Boccaccios Decameron in Hans Sachsens Fastnachtspiel Der gross Eiferer, der sein Weib Beicht höret untersucht. Im Fokus des kontrastiven Vergleichs zwischen der Novelle und dem Fastnachtspiel stehen der Affekt der Eifersucht und das Motiv der List. Die Gründe für die Transformationen im Dramentext von Hans Sachs sind u. a. die Dekontextualisierung der Novelle und ihre Instrumentalisierung als pragmatische Unterweisung der frühbürgerlichen protestantischen Gesellschaft im Bereich der ehelichen Ordnung.
\end{abstract}

Key words: Eifersucht, List, Boccaccio, Decameron, Hans Sachs, Fastnachtspiel, Ehe

Giovanni Boccaccios Decameron, das zwischen 1351 und 1353 geschrieben und im Jahre 1470 zum ersten Mal gedruckt wurde (Frenz 2009, 690), ist gewiss eines der bekanntesten Werke der italienischen Renaissance-Literatur. Es ist eine Sammlung von Erzählungen über die Liebe und andere Themen, die das Leben der Menschen im Spätmittelalter an der Schwelle zur Neuzeit bewegten: „Probleme zwischenmenschlicher Kommunikation, Fragen der Erkenntnis und die Notwendigkeit angemessenen Verhaltens“ (Kocher 2005, 30f.). Wie man der Vorrede (Boccaccio 2008, 9ff.) entnehmen kann, intendiert der Autor mit seinen Geschichten, an Liebeskummer leidende Damen zu trösten und zu erheitern und dadurch ihre Schwermut zu vertreiben. Dies tue er aus Mitleid mit den Betrübten und als Zeichen des Dankes, weil auch er selbst durch die heiteren Erzählungen eines Freundes Trost und Erleichterung gefunden habe. Auch mögen die Damen aus den Geschichten Rat und Belehrung schöpfen. Das Decameron - ein Trost- und Lehrbuch für Frauen? Diese Kategorisierung erweist sich bei genauerer Betrachtung des Textes allerdings als voreilig. Wenn man nämlich noch 
die „dem Buch vorangestellte Stoffanzeige“ (Theisen 1996, 119) - in einer modernen Übersetzung lautet sie: Hier beginnt das Buch, genannt Dekameron, beigenannt Fürst Galeotto (Boccaccio 2008, 7) - mit berücksichtigt, wird die in der Vorrede explizierte Autorintention revidiert. Hinter dieser Namensgebung verbirgt sich eine Anspielung auf Dantes Göttliche Komödie, die die literaturerfahrene LeserIn oder HörerIn erkannt haben musste. Galeotto bzw. Galehaut, in der literarischen Tradition des Mittelalters ein Ritter von Artus' Tafelrunde, war im Lancelot-Roman derjenige, der Ginevra veranlasste, Lancelot zu küssen. Aus dieser Nennung ergibt sich die zu Unrecht negativ konnotierte Interpretation ${ }^{1}$ in manchen Übersetzungen, in denen ,Galeotto' mit ,Erzkuppler' wiedergegeben wird. (Theisen 1996, 119f.) Nach dieser Auslegung wäre das Buch als Anleitung zu unzüchtiger oder freier Liebe zu verstehen. Die dialogisierenden Aussagen schon zu Beginn des Buches können als Signal verstanden werden, das den aufmerksamen Rezipienten zu einem Denkprozess animiert, um den Sinn- oder vielmehr die Sinngehalte des Buches selbstständig zu ergründen.

Charakteristisch ist die Struktur des Werkes, bestehend aus einer Rahmenund 100 Binnenerzählungen. Der Rahmenerzählung zufolge beschließen sieben Damen und drei junge Männer, die der Schicht der Patrizier angehören, zur Zeit der großen Pestepidemie in Florenz, gemeinsam die Stadt zu verlassen und in idyllischer Abgeschiedenheit fern der ihnen drohenden Gefahr, sich durch das Erzählen von Geschichten zu ergötzen. Sie wollen also die Sorgen um ihre Existenz in heiterer Geselligkeit ,vergessen machen', was das ahd. Kausativum irgetzan als Ableitung von irgezzan ursprünglich auch bedeutet, ${ }^{2}$ und sich erfreuen. Die Vielzahl der komischen Erzählungen hat die Intention, Lachen hervorzurufen, das in schweren Zeiten, seinem diätetischen Zweck entsprechend, u. a. als Remedium für Körper und Geist (Jakobs 2006, 176ff.; Arend 2004, 108 ff.) fungieren soll, wenn auch der didaktische Aspekt des Komischen (vgl. Suchomski 1975) nicht vernachlässigt werden sollte. Jedoch kann der Rezipient diesen Geschichten keine eindeutig formulierte Lehre entnehmen. Er muss sie vielmehr durch eigenständige Reflexion aus der jeweiligen Erzählung im Zusammenspiel mit der Gesamtheit der sie betreffenden Kommentare des extradiegetischen oder der intradiegetischen Erzähler oder deren Reaktionen selbst erschließen, wobei aber die Aussagen, die ihm eine Orientierung bieten, einander widersprechen können und sich dadurch dekonstruieren. (Vgl. Kocher 2005, 31, 153ff.)

Dass Boccaccio mit seinem Decameron die ,Novelle' als neue Erzählgattung mit spezifischen Strukturmerkmalen geschaffen habe, gilt in der Forschung jetzt allgemein als obsolet, da sich seine Geschichten von anderen traditionellen kürzeren Erzählformen, wie Fabeln, Mären, Anekdoten und Schwänken, nicht wesentlich unterscheiden (vgl. Bolsinger 1998, 15ff.; Kocher 2005, 35), hat es doch damals normierte Gattungsbezeichnungen im heutigen Sinne nicht gegeben. Gattungsbestimmend sind nach Hans-Robert Jauß (1977, 34ff.) indes die verschiedenen Aspekte der kommunikativen Situation, das Verhältnis zur Tradition und der ,Sitz im Leben'. Eines der entscheidenden, im Nachhinein auch für die frühe ,Novellistik' geltenden Konstitutionsmerkmale besteht nach Wehle darin, „daß ihre einzelnen

\footnotetext{
${ }^{1}$ Morf spricht von ,ritterlich feine[m] Liebesbote[n]“, zit. n. Theisen 1996, 119.

${ }^{2}$ Vgl. Duden. Etymologie 1963, 142: ,,woraus sich seit dem 15. Jh. der Sinn , sich erholen, [sich] erfreuen' entwickelte.“
} 
Geschichten ebenso wie ihre Sammlungen, die sie vereinigen, mit Beharrlichkeit das Erzähltwerden als eine ihrer Grundvoraussetzungen demonstrieren“" (zit. n. Bolsinger 1998, 17). Konstitutiv für die Gattung der Novelle ist demnach die Erzählsituation, aus der die Selbstreferentialität der Texte resultiert - und darin liege die Modernität von Boccaccios Novellensammlung. (Bolsinger 1998, 16f.)

Das Decameron, das aufgrund „seiner literarischen Qualität“ (Theisen 1996, 116) heute zur Weltliteratur zählt, stieß in Deutschland zunächst auf geringe Resonanz. Anfänglich waren wohl nur die lateinischen Versionen einzelner Novellen in Humanistenkreisen bekannt (Dallapiazza 1987; Bertelsmeier-Kierst 1998; Münkler 2004, 85f.). Einen breiteren Zugang zum vollständigen Text erlangten die literarisch Interessierten dagegen erst durch die erste deutsche Gesamtübersetzung eines gewissen Arigo, dessen Identität bis heute nicht gänzlich geklärt ist. ${ }^{3}$ Zum ersten Mal erschien sie um 1476 bei Zainer in Ulm, der zweite Druck von 1490 bei Anton Sorg wurde mit Holzschnitten versehen, und 1535 erschien in Straßburg der sog. CammerlanderDruck, mit dem eine breitere Wirkung im deutschen Sprachraum einsetzte. Daneben kursierten die in den Jahren 1509 und 1519 gedruckten gekürzten Ausgaben, die moralisierende, rezeptionslenkende Vierzeiler enthielten. (Dallapiazza 1991, 181; ders. 2012, 87) Bei der Übertragung ins Deutsche verzichtete Arigo zwar weitgehend auf kulturelle ,Eindeutschungen' (vgl. Theisen 1996, 116), passte aber den Text an die im 15. Jahrhundert vorherrschende Erzählerwartung der urbanen Gesellschaft in Deutschland an, indem er im Sinne einer Tugend- und Morallehre die Mehrdeutigkeit der Diskurse reduzierte. Dies hatte einen Verlust der poetologischen Komplexität zur Folge (vgl. Bolsinger 1998, S. 153 u. Münkler 2004, 89f.) $)^{4}$. Im Vergleich zur ersten Ausgabe von $1476 \mathrm{nahm}$ in den folgenden deutschen Ausgaben die Moralisierung sogar noch zu, da in einigen Ausgaben, wie oben erwähnt, am Ende der Novellen ein Epimythion hinzugefügt wurde. In der Ausgabe von Cammerlander aus dem Jahre 1535 kam es zu weiteren Veränderungen. Der Text wurde sprachlich überarbeitet (vgl. u. a. Meid 2006, 94) und wie Claudia Bolsinger $(1998,155,20)$ feststellt, wurden „die schwankhaften Elemente herausgefiltert“, „Schluß, Einleitung und Rahmenhandlung weiter gekürzt". Auf diese entstellte und purgierte Ausgabe griffen nun die meisten Autoren zurück, die Boccaccios Decameron als Vorlage für ihre produktive Rezeption benutzten (Dallapiazza 1991, 181).

Einer der zahlreichen Bearbeiter von Boccaccios Novellen war der Nürnberger ,Schusterdichter' Hans Sachs. Er war der produktivste und einer der vielseitigsten Autoren des 16. Jahrhunderts (Hahn 1993, 409). Nach Angabe von Dallapiazza $(2012,92)$ konnten bislang 128 von Sachsens Spruchgedichten bzw. Dramen und 79 Meisterlieder ermittelt werden, die auf Boccaccios Decameron zurückgehen. ${ }^{5}$ Sein literarisches Schaffen ist eng verbunden mit der streng hierarchisch gegliederten freien Reichstadt Nürnberg, die zur Zeit von Hans Sachs ein prosperierendes und geistigkulturelles Zentrum war (Brunner 1976, 1ff.). Als Handwerksmeister gehörte er der

\footnotetext{
${ }^{3}$ Ausführlicher dazu Theisen 1996, 1ff., vgl., auch Kocher 2005, 22.

${ }^{4}$ Zur detaillierten komparatistischen Analyse siehe Theisen 1996.

${ }^{5}$ Hartmann $(1911,22)$ ging noch von 51 Meistergesängen, 31 Spruchgedichten, 13 Fastnachtsspielen, 6 Komödien und 2 Tragödien aus, die Sachs nach der Vorlage der deutschen Decameron-Übersetzung verfasste. Hartmanns Ermittlungen zufolge dienten Hans Sachs als Quelle für seine literarische Produktion 62 Novellen und die Rahmenerzählung der vierten Tagreise. Isenring $(1962,53)$ erhöhte die Zahl der Spruchgedichte auf 33.
} 
Mittelschicht an, gelangte zu materiellem Wohlstand, so dass er sich schließlich allein seiner literarischen Produktion widmen konnte. Er hatte aber kein politisches Mitspracherecht (Könneker 1991, 99). Zum Adressatenkreis seiner Literatur gehörten die städtischen Mittel- und Unterschichten, an deren Erwartungshorizont und Kommunikationsbedingungen er die Inhalte und Funktionen seiner dichterischen Praxis orientierte. Ein wesentliches Merkmal der damaligen literarischen Kommunikation war die gemeinschaftliche Rezeption von Literatur, die neben ihrem unterhaltenden Aspekt bekanntlich die Funktion hatte, Wissen und an den Normen orientierte praktische Handlungsanweisungen zu vermitteln. Hans Sachs war ein großer Verfechter Luthers und der Reformation und propagierte in seinen Werken eine dem Gemeinwohl nutzende bürgerlich-protestantische Sittenlehre. (Hahn 1993, 410ff.; Kugler 2004, 412) Dies brachte Sachs den in der Forschung dominierenden Vorwurf ein, dass er ,an sämtl. Stoffe den Maßstab einer vergleichsweise eng gefaßten bürgerlich-protestantischen Moral anlegte, die im Epilog - wie meist auch in der Schlußsentenz seiner übrigen Texte [d. h. nicht nur dramatischen Texte, Anm. MJB] - auf eine einprägsame Formel gebracht wird.“ (Könneker 1991, 101f.)

Auf der Grundlage eines intertextuellen Vergleichs von Boccaccios fünfter Novelle des siebten Tages in der deutschen Arigo-Übersetzung und des Fastnachtspiels Der groß Eyferer, der sein Weib Beicht höret ${ }^{6}$, das Hans Sachs (1883, 89-101) im Jahre 1563 verfasste, soll im Folgenden untersucht werden, inwiefern beim produktiven Rezeptionsakt die Ausgangstexte modifiziert wurden und worin die Gründe für die Transformationen bei den ästhetischen und kulturellen Grenzüberschreitungen zu suchen sind. Als Vergleichsgrundlage soll in erster Linie der Cammerlander-Druck von 1535 dienen, auf den Hans Sachs wohl zurückgegriffen hat (vgl. Dallapiazza 2012, 87). Ergänzend dazu wird die Druckfassung von 1476 herangezogen, die im Vergleich zur späteren Ausgabe durch die vollständigere Rahmenerzählung und eine größere inhaltliche Nähe zu Boccaccios Decameron die ursprüngliche Kontextualisierung der Novelle eher aufzeigt und dadurch eine Ausgangsbasis für die Interpretation des Textes im Erzählzusammenhang der Novellensammlung bietet.

Das siebte Kapitel der Novellensammlung ist Erzählungen gewidmet, die von der Listigkeit der Frauen handeln. Überschrift und Rahmenerzählung sind im Cammerlander-Druck zu folgendem sachlich-knappen Text verschmolzen:

\section{Die Siebent Tagreyß.}

DArinn wirt die erber gesellschafft sagen von den frawen / die vmb liebe vnd beschutzung ihrer ehr / ir männer züchtiglichen betrogen haben / dann sie eynes solchen nit wargenummen. Da nu der tag anbrach / gebot die [!] künig Emilie / der vorgesagten materie eyn anfang zu geben $\left(\right.$ Camerlander, cxxxviiir ${ }^{7}$

\footnotetext{
${ }^{6}$ In der Überschrift und in den folgenden Zitaten werden die Grapheme für Umlaute der modernen Schreibweise angepasst. Das sog. „Schaft-s“ wird durch rundes $s$ ersetzt. - Zur produktiven Rezeption der Novelle VII, 5 bei Hans Sachs in den verschiedenen Gattungen vgl. Dallapiazza 2011, 473f. u. ders. 2012, 100f.

${ }^{7}$ Bei der Transkription der Zitate werden die Grapheme der modernen Schreibweise angepasst und die Abkürzungen aufgelöst.
} 
Diese nüchtern konstatierende, eher wertneutrale Äußerung unterscheidet sich erheblich vom suggestiven Wortlaut des Zainer-Drucks, der die Deutung der folgenden Erzählungen erheblich beeinflusst und in eine positive Richtung lenkt:

\section{GOT VNS DIE SIBENDEN ZUO GUOTEM END WEND.}

Vnder dem gewalte vnd regiment des künigs Dioneo dise wirdig geselschaft sagen wirt von den frawen die vmb liebe vnd beschüczung irer eren willen ire mann betrogen haben, vnnd die solicher betrügung nit war genomen noch die erkannt haben, vmb des willen die frawen bei iren eren beliben sein. (Zainer, 409)

Dieses heikle, moralisch eher fragwürdige Thema wird in der Erzählgemeinschaft nicht diskussionslos hingenommen. Es ruft Einwände von Seiten der züchtigen frawen (Zainer, 407) hervor, die es nicht schicklich finden, diesen Gegenstand zu behandeln und die Dioneo, den gewählten König des Tages, bitten, er möge doch ein anderes Thema wählen. Dioneo beruft sich aber dezidiert auf die uneingeschränkte Redefreiheit in einer Zeit der existentiellen Bedrohung, in der alle weltlichen und geistlichen Gesetze aufgehoben sind und in der jeder nach seinem Gefallen handeln solle, nur um sein Leben zu schützen. Die etwas unsittlichen Erzählungen allein seien, solange man sie nicht in Werke umsetze, nicht schädlich. Ihr Zweck sei es, Freude zu spenden, ohne eine Bestrafung zu fürchten. Dann fährt er ferner fort, dass sich eine der jungen Damen eher kompromittieren würde, sollte sie sich weigern, über ein solches Thema zu sprechen, weil sie gerade dadurch in den Verdacht geriete, selbst mit solchen Sünden beladen zu sein. (Zainer, 407f.) Das Rahmenthema und die kontroverse Debatte über Wahrung des Dekorums und die Lizenzierung des Anzüglichen bilden also den Kontext, in den die Novellen des siebten Tages zunächst eingebettet sind, und müssen als Interpretationsfolie Berücksichtigung finden.

Die besagte Novelle (Camerlander cxliiiir-cxlviv ${ }^{\mathrm{r}}$, Zainer 427-434) handelt von einem Mann, der auf seine Frau grundlos eifersüchtig ist. Er bewacht sie ohne Unterlass und verbietet ihr jeglichen gesellschaftlichen Kontakt außerhalb des Hauses. Selbst an Sonn- und Feiertagen darf sie nicht in Gesellschaft verkehren und Freude daran haben. Der unbegründeten Verdächtigungen und Terrorisierungen durch den Ehemann überdrüssig, beschließt die Frau, seine Beschuldigungen mit ihren Taten zu rechtfertigen und sich einen Liebhaber zu suchen. Da sie von der Außenwelt abgeschirmt ist, hat sie keine große Auswahl. In ihrer Not erinnert sie sich an den jungen Mann, der im Nachbarhaus wohnt. Mit ihm nimmt sie Kontakt auf, indem sie ein Loch in die Wand seines anliegenden Zimmers gräbt, so dass sie sich näherkommen. Als sie zu Weihnachten ihren Gatten um Erlaubnis bittet, zur Beichte zu gehen, schöpft er noch mehr Verdacht und ist begierig, ihre Sünden zu erfahren. Weil sie sich weigert, ihm ihre Sünden zu offenbaren, da er ja kein Priester sei, beschließt er, sich als Kaplan zu verkleiden und ihr in der Rolle eines Geistlichen die Beichte abzunehmen. Trotz der Maskerade erkennt die Frau ihren Mann und entschließt sich im Nu, mit Worten seinen Verdacht zu bestätigen. In der Beichte gibt sie vor, einen Pfaffen zu lieben, der jede Nacht bei ihr schlafe. Nur aus Begierde, mehr darüber zu erfahren, zügelt der Eyferer 
seine Erregung und fragt die Frau, wie der Pfaffe denn bei ihr liegen könne, wenn doch der Ehemann im Hause sei. Sie spielt auf ihr unbekannte Künste an, mit deren Hilfe der Pfaffe die Türen öffne und den Ehemann in den Schlaf wiege. Der ,Beichtvater' weist sie auf die Unrechtmäßigkeit ihres Handelns hin und fordert sie auf, die Beziehung abzubrechen. Doch die Frau weigert sich, weil sie, wie sie sagt, den Pfaffen zu sehr liebe. Unter diesen Umständen könne der Pfaffe ihr zwar keine Absolution erteilen, er wolle aber ihr zuliebe für sie beten und einen Kleriker zu ihr schicken, damit er ihm mitteilen könne, ob seine Gebete geholfen hätten. Nach einer solchen unerhörten ,Entdeckung' kann der Mann zu Hause seine Aufgebrachtheit nicht verbergen. Er setzt seinen Plan, die Beziehung zwischen seiner Frau und dem unbekannten Pfaffen zu unterbinden, in die Tat um. Er gibt vor, die nächste Nacht - allerdings ohne Angabe des Grundes - woanders verbringen zu müssen, und erteilt seiner Frau Anweisungen, dass sie während seiner Abwesenheit alle Türen zu versperren habe. Nachdem der Mann weggegangen ist, eilt sie zu dem Loch in der Wand, um ihrem Liebsten ein Zeichen zu geben, dass er zu ihr kommen könne. Während die beiden eine erfüllte Liebesnacht miteinander verbringen, liegt der Mann in voller Ausrüstung in einer Kammer auf der Lauer, begierig, den Pfaffen zu erhaschen und zu züchtigen. Doch der Eifersüchtige hat, hungrig und frierend, vergebens gewartet und legt sich schlafen. An diesem Tag schickt er einen Schüler zu seiner Frau, der sie, wie vereinbart, nach dem Pfaffen fragen solle, ob er denn noch käme. Die Frau, welche die Bosheit des Mannes erkennt, sagt, er sei vergangene Nacht nicht gekommen. Wenn er fernbliebe, könnte sie ihn noch vergessen, doch das wolle sie ganz und gar nicht. Der Mann steht noch viele Nächte Wache, während sich seine Frau mit dem Geliebten vergnügt. Des vergeblichen Wachens schließlich müde, fragt der Mann die Frau, was sie dem Pfaffen damals gebeichtet habe und warum der Kleriker so oft zu ihr komme. Die Frau weigert sich zu antworten, er in Rage - beschimpft sie du zunichtes böses weib! (Cammerlander, cxlvir) und gibt ihr schließlich zu erkennen, dass er davon unterrichtet sei. Sie solle ihm die Identität des Pfaffen preisgeben, wenn nicht, droht er sie zu töten. Die Frau klärt ihn nach etlichen Verzögerungen in allen Details über den Sachverhalt auf, dass sie keinen Pfaffen liebe, sondern ihn bei der Beichte erkannt habe, und fährt fort mit den Worten:

Nun was zunichten mannes magstu nur sein / das du dich die falschen vntugent des eiffern hast vberwinden vnd so schentlich blenden lassen I (Cammerlander, cxlvi ${ }^{\vee}$ )

Er solle Einfalt und Eifersucht ablegen, zu einem Mann werden, damit er nicht zum Gespött der Leute werde, die seine Torheit bemerken. ${ }^{8}$ Sie schwört ihm bei Gott und den Heiligen, wenn es ihr in den Sinn käme, ihm Hörner aufzusetzen, wäre sie fähig - auch wenn er hundert Augen hätte - ihn zu blenden und ihn ohne sein Wissen zu betrügen. Der Mann schämt sich wegen seines Verhaltens, sieht die Sinnlosigkeit seiner Eifersucht ein und hält - welch Paradox - von da an seine Frau in Ehren, obwohl er jetzt, da sie tatsächlich einen Geliebten hat, Grund zur Eifersucht hätte.

\footnotetext{
${ }^{8}$ Ich sag dir das du ab von deiner einfeltigkeit lassest/vnd würd zu einem man [Hervorhebung von der Verf., MJB] / las dein eiffern vnd bös gedencken / damit du nit jedermanns gespött seiest / die dein thorheyt vernemmen. (Cammerlander, cxlvivi $)$
} 
Das Hauptmotiv der Novelle ist also die Eifersucht. Wohin dieser Affekt, der - da er den Mann der Vernunft beraubt - als , weibisch' markiert wird, führen kann, wird anschaulich demonstriert und im einleitenden Erzählerdiskurs des Zainer-Drucks (428) angekündigt: zu Übel und Verderben des Eifersüchtigen, zumal die Eifersucht nicht gerechtfertigt ist. Die Frau hat sich nichts zu Schulden kommen lassen, allein seine übermäßige Liebe und die Bereitschaft seiner Frau, ihm stets zu Willen zu sein, sind Anlass zu seiner übertriebenen Angst vor Liebesrivalen. Fehlendes Maß und Ausschaltung seiner Vernunft führen u. a. zum Verlust der Fähigkeit, die Realität richtig einzuschätzen. Der Eifersüchtige ist in seiner Erkenntnis begrenzt. Geblendet durch den Affekt, weiß er die Antworten der Frau im Beichtstuhl nicht richtig zu deuten und glaubt sogar an das Irrationale, als sie ihm von gewissen Künsten des Pfaffen berichtet, mit denen dieser sich bei ihr Einlass verschaffe und ihn, den Gatten, in Schlaf versenke.

Nicht nur sein Geist ist getrübt, die Eifersucht schadet auch seinem physischen Wohlbefinden und der aus seinem Affekt resultierende Zorn steigert sein Gewaltpotential gegenüber dem Nebenbuhler und seiner Frau:

Der eifferer in seinem vnglück geschwollen auffstund / des pfaffen gewandt gieng außzuziehen / vnd sich heym zuhauß fügt. Vnnd bedencken ward wie er den pfaffen bei dem weib begreiffen möcht / damitt er einem vnd andern böß spiel zurichten möcht. (Cammerlander, $\mathrm{cxlv}^{v}$ )

Der Ehemann terrorisiert seine Frau, die sehr unter seinem Regiment leidet. Er befolgt weder die weltlichen noch die göttlichen Gesetze, wenn er ihr nicht einmal am siebten Tag der Woche Freude und Ruhe vergönnt - zur Ehre des Schöpfers und zu ihrer eigenen Regeneration. Das Ausmaß ihres Leidens wird hinlänglich geschildert.

Hätte sich der Mann von der Vernunft leiten lassen, so hätte er seine rational nicht nachvollziehbare Eifersucht gezügelt. Die daraus schließende Notwendigkeit, die Affekte zu bändigen, ist ein Reflex auf die vom Stoizismus beeinflusste RenaissancePhilosophie, deren Vertreter Petrarca (Meuer 2008) mit Boccaccio auch eine enge Freundschaft verband (Kocher 2006). Doch scheint sich die Verbindung von der Beherrschung der Eifersucht mit der daraus resultierenden Erkenntnis der Welt im Schluss der Erzählung, als die Frau ihn tatsächlich betrügt, er ihr aber vollstes Vertrauen entgegenbringt, aufzuheben.

Ein zweites Motiv ist die listige Frau. Dank ihrer Schlauheit gelingt es ihr, durch ihre Handlungsweise den Mann eines Besseren zu belehren. Sie führt ihn hinters Licht, indem sie, ohne dass er es durchschaut, bei seiner Inszenierung der Beichte die ihr zugewiesene Rolle spielt und ihn, um sich zu rächen, noch provoziert. Schließlich dient ihr die Wahrheit als probates Mittel, ihren nun geläuterten Ehemann zu blenden, um die Freuden der außerehelichen Liebe nach Lust und Laune zu genießen:

Ich sprich vnd schwer dir des zu got vnd allen heiligen / kem es in meinen sinn dir dy hörner zu machen vnd auffsetzten vnd hettestu hundert augen / als du zwei hast / ich solte dich bei in allen blenden / vnnd meinem willen eyn genügen thun on dein wissen. (Cammerlander, cxlvivi 
Ihr Vorgehen, ihren Mann zu überlisten und sich aus Genugtuung einen Liebhaber zu nehmen, erscheint durch den einleitenden Erzählerkommentar im ZainerDruck und die Schilderung der Ausmaße ihres Leidens gerechtfertigt. Ihr Verhalten wird als Notwehr, ent-schuldigt'.

vnd [...] gedencke mir was übels sölichen eyferern zustet, sunder wenn sy on vrsache in söliche eyfern fallen in wol an ste vnd in keynen weg zu klagen seyen, vnd das die meister der gesecze alle gesecze halten sölten so spreche ich wol das sie den frawen kein ander pen sölten geben haben, dann das sie den geben haben die wider die gesecze thon sich selbes zu beschüczen, Dann die eyferer der iungen frawen leben zerstörer sein vnd fleißig sucher ires todes (Zainer, 428)

Doch wird das auf der Ebene des Erzähldiskurses legitim erscheinende weibliche Handeln durch die kontroverse Debatte am Ende der sechsten Tagreise (Zainer, 407f.) ${ }^{9}$ relativiert, in der ausdrücklich zwischen Erzählen und das Erzählte in die Tat umsetzen differenziert wird. Die fünfte Novelle des siebten Tages in diesem Erzählkontext bedingungslos als Anleitung für die Auflehnung der Frauen auszulegen, die aufgrund der unbegründeten Eifersucht ihrer Männer leiden, ist demnach nicht möglich. Die Fiktion bietet also - im Unterschied zur ,realen' Welt - einen Freiraum, moralische Schranken zu überschreiten und Handlungen, die den ethischen Normen widersprechen, ohne Konsequenzen , auszuprobieren' und sich von den Zwängen der gesellschaftlichen Ordnung zumindest während des Erzählens bzw. der Rezeption der Erzählung zu befreien. Im Cammerlander-Druck ist diese Diskussion über die Zulässigkeit des vorgeschlagenen, am siebten Tag zu behandelnden Themas nicht vorhanden. Die Erzählungen der siebten Tagreiß schließen sich nahezu kommentarlos der letzten Novelle des sechsten Tages an, dennoch findet sich in beiden Versionen zu Beginn der folgenden sechsten Novelle ein zustimmender Kommentar des Rahmenerzählers. Im Cammerlander-Druck (cxlviv) begrüßt er die Bestrafung des eifersüchtigen Ehemannes und setzt die LeserIn vom bejahenden Lachen der weiblichen Zuhörerschaft in Kenntnis, in der Zainer-Ausgabe (435) erfährt man, dass die Geschichte sowohl bei den Männern als auch den Frauen Gefallen findet und dass sie das Vorgehen der Frau gutheißen.

Die Novelle vom eifersüchtigen Ehemann beginnt im Zainer-Druck (428) mit der Reflexion der Erzählerin über die Problematik der Eifersucht. Diese Überlegungen haben eine wertende und voraus weisende Funktion. Dagegen sind im CammerlanderDruck, wie schon erwähnt, die Kommentare stark eingeschränkt, wodurch der wenn bisweilen auch ambivalente Kontext, der eine gewisse Orientierungsfunktion bei der Deutung der Novellen durch die LeserIn ausübt, erheblich reduziert ist. In beiden Fassungen schildert die auktoriale Erzählerin auf der Ebene des Erzähldiskurses den Handlungsverlauf in einem chronologisch-kausalen Zusammenhang, so dass dieser

\footnotetext{
${ }^{9}[. .$.$] ob ir in eüerm reden ein kleyn mynder dann czüchtig seit schat nicht, nun solichem in wercken nicht$ nachkomet, dann wir das thun vns freüd czegeben, darumb kan ich ye nicht vernämen mit was widerrede oder argument ir von yemand mügt gestraft werden über daz was bis auf disen heutigen tag ist gesaget worden [...]Ich nichte vernämen mage, das wir darumb in keinerlei czestraffen sein, noch das hinfür mit der hilf gotes geschehen sol. (Zainer, 408)
} 
im Wesentlichen linear fortschreitet. Die Retardierung des Handlungsverlaufs an einigen Stellen ${ }^{10}$ bewirkt eine gewisse Steigerung der Spannung. Die Beweggründe und Empfindungen der handelnden Personen werden zum Teil aus der Außenperspektive durch die Erzählerin oder aus der Innenperspektive der Personen in Dialogen vermittelt.

Hans Sachsens Fastnachtspiel Der groß Eyferer, der sein Weib Beicht höret mit vier Personen und einem Akt, der in 19 implizite Szenen unterteilt ist, hält sich zwar in Umrissen an die Vorlage, bringt aber einige sinnträchtige inhaltliche und formale Unterschiede ein. Eine wichtige Differenz besteht zunächst darin, dass Sachs die Handlung aus dem vorgegebenen Rahmen isoliert (vgl. u. a. Dallapiazza 2012, 99). Dadurch ist die auf der Kontextualisierung basierende Mehrdeutigkeit der Novellen aufgehoben. Der Interpretationsprozess erfolgte beim Fastnachtspiel ursprünglich auf der Grundlage der Inszenierung in einer normorientierten städtischen Zuschauergemeinschaft.

Das Motiv der unbegründeten Eifersucht ist hier anders akzentuiert. Es geht nicht so sehr um die Zurschaustellung der Erkenntnishemmung infolge des Affektes, denn der Ehemann ist sich dessen durchaus bewusst, dass er eigentlich keinen Grund zur Eifersucht hat (HS, vv. 67f.). Im Zentrum des Fastnachtspiels steht vielmehr die Demonstration des aus dem Affekt resultierenden Zornes, einer der sieben Hauptsünden (ira) (Hedwig 2002), die den Menschen zu unüberlegten Gewaltaktionen in Worten und Werken gegenüber seinen Nächsten veranlasst. Belege für die Gewaltbereitschaft des Mannes gibt es mehrere, in einem Monolog äußert er sich beispielsweise folgendermaßen:

Nun wil ich nab, sammer botz jammer!

Am Thennen stehn in die Holtzkammer

Vnd auff den Pfaffn haben mein spür.

Sperrt er auff, schleicht rein durch die thür,

Ich wil jn schlagen auff sein Platten.

Solt ich verschlagn hundert Ducaten

Vnd kommen gleich in schweren Bann,

Doch ich jms nit vertragen kan;

Ob ich den Schelm gleich zu todt schlag,

Ich in vor tags ins Wasser trag

Vnd laß jn schwimmen den Ehrndieb

Wenn er nur heint nit aussen blieb! (HS vv. 212-223)

Ein solches Handeln hat, aus religiöser Perspektive betrachtet, verhängnisvolle Folgen für das Heil des Mannes, bedroht aber auch seine bürgerliche Existenz. Als Gewalttäter hat der Eifersüchtige mit Strafverfolgung und Ausschluss aus der Gemeinschaft zu rechnen. Allein die Verschwendung des Geldes, wovon im obigen Zitat auch die Rede ist, wirkt sich für die Existenz eines Bürgers nachteilig aus, hat doch nach damaligen bürgerlich-protestantischen Vorstellungen der Mann u. a. für das materielle Wohlergehen seines Hausstandes Sorge zu tragen. (Luther 1828, 179ff.) Darüber hinaus steht schon

\footnotetext{
${ }^{10}$ Z. B. rhetorische Frage als handlungsverzögerndes und spannungssteigerndes Moment (Zainer, 433; Cammerlander, cxlcir $^{\mathrm{r}}$.
} 
in der Bibel, dass der Mann nicht zu eifersüchtig über seine Frau wachen dürfe, weil er sie sonst provoziere, ihm Böses anzutun (Sirach 9,1). In Sachsens Fastnachtspiel macht sich die Frau allerdings keines Vergehens schuldig, sie bedient sich lediglich einer List, um ihren Gatten auf den Pfad der Tugend zu führen.

Dass die Frau die List bei der Beichte mit gutem Grund anwendet, kann man u. a. ihren Monologen entnehmen, wo sie über die Ausmaße ihres Leidens spricht (z. B. HS, vv. 1-19), das ihr der keineswegs makellose Ehemann zufügt, der sie nicht nur grundlos der Untreue bezichtigt, sondern der sie bisweilen selbst betrügt, als unzüchtiges Weib beschimpft, rauft und schlägt ${ }^{11}$. Mit der List beabsichtigt sie ihren Mann um der Besserung willen in der Öffentlichkeit bloßzustellen. Er muß ziehen im Eselskarren (HS 265), sagt sie an einer Stelle und spielt wohl auf einen Rügebrauch ${ }^{12}$ an. Im Unterschied zur Frau der obigen Novelle bleibt sie ihrer Erziehung treu und besteht bis zum Ende darauf, sich nicht zu kompromittieren und in ihrer Ehre standhaft zu bleiben, obwohl sie immer wieder von ihrer Magd animiert wird, sich einen Liebhaber, anzulachen'. So verkörpert sie eine vorbildliche Ehefrau, die ihren Partner in die Schranken weist, um die eheliche Harmonie wieder herzustellen, da für sie im Sinne von Hans Sachs, dessen vom Protestantismus beeinflusste Überzeugung es war, „daß die Ehe trotz ihrer offenkundigen Mängel als soziale [...] Institution eine von Gott gebotene Lebensform ist, der sich ungestraft niemand entziehen kann“ (Könneker 1976, 228), eine Scheidung nicht in Frage kommt. Das Fastnachtspiel endet mit dem Auftritt des Mannes, der seine Frau um Vergebung bittet, weil er ihre Ehrenhaftigkeit erkannt hat, und ihr in Zukunft sein Vertrauen schenken will (HS 333-337).

Hervorzuheben ist, dass sich Hans Sachs einer expliziten ,Moral' enthält (vgl. Dallapiazza 2012, 101). Diese ist hier auch nicht notwendig, denn durch die Inszenierung der Problematik, den positiven Wandel des Mannes und der Integrität der Frau können die Zuschauer selbst nachvollziehen, wie sich die Menschen verhalten sollen und Konflikte zu lösen sind, damit die gesellschaftliche Ordnung wieder hergestellt und die Gemeinschaft funktionsfähig ist. In dieser Hinsicht ist das Fastnachtspiel eindeutig, aber eine Lehre wird den Rezipienten nicht aufoktroyiert. Sie können sie vielmehr selbst durch eigene Interpretationsleistung formulieren.

Aufgrund des Gattungswechsels kommt es u. a. zu Unterschieden in der Struktur der Handlung. Sie verläuft nicht mehr linear, sondern wird durch Rückblicke und Vorausdeutungen unterbrochen. Die Außenperspektive des auktorialen Erzählers weicht hier einer Innenperspektive der handelnden Personen, die ihre Lage, Beweggründe und Erklärungen in Dialogen und Monologen kundtun. Gattungsbedingt ist auch die größere Rolle der Magd, die als Gesprächspartnerin, zum Teil die Funktion der Erzählerin in der Novelle übernimmt, die den Rezipienten über die Zusammenhänge aufklärt und die Handlung kommentiert. Dem Fastnachtspiel entsprechend wählt der Autor oft eine volkstümlichere Sprache, als sie in der Vorlage zu finden ist. Kennzeichnend dafür sind die zum Teil spöttisch-grobianische Ausdrucksweise (z. B. HS, vv. 105f., 212ff., ). Formale Merkmale der Dichtung sind ferner Knittelvers und Paarreim.

\footnotetext{
${ }^{11}$ HS, v. 288: Mich huret, säcket, raufft vnd schlug. - Das Verb säcken kann interpretiert werden als einen sack schelten (vgl. DWB Bd. 8, 1893, Sp. 1622), wobei sack u. a. ein Schimpfwort „für faule und besonders für unzüchtige weiber" ist (DWB Bd. 8, 1893, Sp. 1616).

${ }^{12}$ Vgl. dazu die Erklärung zu Karrenziehen als Ehrenstrafe in DWB Bd. 5, 1873, Sp. 229. Zu den Rügebräuchen vgl. u. a. Röcke 2005, 63.
} 
Die vergleichende Analyse hat ergeben, dass sich die inhaltlichen und formalen Unterschiede zum einen aus der Anbindung des Stoffes in den Gebrauchszusammenhang der Fastnachtspiele und deren Funktion in der städtischen, protestantisch geprägten Gemeinschaft der Frühen Neuzeit und zum anderen durch den Gattungswechsel vom erzählenden zum dramatischen Text ergeben. Die ambivalente Bedeutung von Boccaccios Novelle in den frühneuhochdeutschen Fassungen, die auf der Kontextualisierung des Textes in einem größeren Erzählzusammenhang gründet, wird in Hans Sachsens Fastnachtspiel zugunsten einer unmissverständlichen Belehrung aufgegeben, auch wenn diese nicht explizit formuliert ist. Während in der Novelle VII, 5 die Eifersucht des Mannes vornehmlich mit mangelnder Erkenntnisfähigkeit in Verbindung gesetzt wird, wird dieser Affekt bei Sachs durch die pointierte Bezugsetzung zum Zorn als Sünde auf die religiöse Ebene transponiert. Die List dient im Fastnachtspiel aufgrund der moralischen Integrität der Frau ausschließlich dazu, den Mann von der Eifersucht zu kurieren und die von Gott gegebene eheliche Ordnung zu restituieren, während sie in der Novelle der Frau die Möglichkeit bietet, ihren Mann in der Tat zu betrügen. Wie ihr Handeln zu werten ist, bleibt letztendlich der LeserIn überlassen.

\section{Universität Ljubljana, Slowenien}

\section{LITERATURVERZEICHNIS}

\section{QUELLEN}

Die Bibel. Nach der Übersetzung Martin Luthers. Mit Apokryphen. Stuttgart: Deutsche Bibelgesellschaft 1985.

Boccaccio, Giovanni: Das Dekameron. Nach der Übertragung aus dem Italienischen von Karl Witte. Frankfurt am Main: Fischer Taschenbuch Verlag 2008.

Centum novella Johannis Boccacii. Straßburg: Cammerlander 1535. Exemplar der Herzog-AugustBibliothek, Wolfenbüttel, Sign. 155.2 Quodl. (2). (Cammerlander)

Decameron von Heinrich Steinhöwel [!]. Hrsg. von Adelbert von Keller. Stuttgart: Litterarischer Verein 1860. (Zainer)

Luther, Martin: „Predigt von dem Ehestande, gehalten zu Wittenberg. Anno 1525“. Dr. Martin Luthers sämmtliche Werke. Bd. 16, 1. Abt.: Homiletische und katechetische Schriften. Erlangen: Verlag von Carl Heyder 1828, 165-189.

Sachs, Hans: „45. Ein Faßtnachtspiel mit 4 Personen vnd wird genennet: Der groß Eyferer, der sein Weib Beicht höret“. Elf Fastnachtspiele aus den Jahren 1552 und 1553. Hrsg. von Edmund Goetze. Halle: Max Niemeyer 1883, 89-101.

\section{FORSCHUNGSLITERATUR}

Arend, Elisabeth: Lachen und Komik in Giovanni Boccaccios Decameron. Frankfurt am Main: Vittorio Klostermann 2004.

Bertelsmeier-Kierst, Christa: „Wer rezipiert Boccaccio? Zur Adaptation von Boccaccios Werken in der deutschen Literatur des 15. Jahrhunderts. ZfdA 127 (1998), 410-426. 
Bolsinger, Claudia: Das Decameron in Deutschland. Wege der Literaturrezeption im 15. und 16. Jahrhundert. Frankfurt / Berlin / Bern / New York / Paris / Wien: Peter Lang 1998.

Brunner, Horst: „Hans Sachs - Über die Schwierigkeiten literarischen Schaffens in der Reichsstadt Nürnberg“. Horst Brunner, Gerhard Hirschmann u. Fritz Schnelbögl (Hgg.): Hans Sachs und Nürnberg... 1-13.

Brunner, Horst, Gerhard Hirschmann u. Fritz Schnelbögl (Hgg.): Hans Sachs und Nürnberg. Bedingungen und Probleme reichsstädtischer Literatur. Hans Sachs zum 400. Todestag am 19. Januar 1976. Nürnberg: Selbstverlag des Vereins für Geschichte der Stadt Nürnberg 1976.

Dallapiazza, Michael: „’Decamerone’ oder ,De claris mulieribus'? Anmerkungen zur frühesten deutschen Boccaccio-Rezeption, ZfdA 116 (1987), 104-118.

"Die Bedeutung Nürnbergs für die frühe deutsche Boccaccio-Rezeption. Volker Knapp u. Frank-Rutger Hausmann (Hgg.): Nürnberg und Italien: Bedingungen, Einflüsse und Ideen. Tübingen: Stauffenburg-Verlag 1991, 181-193.

„In Cento novella man list. Boccaccios 'Decamerone' in den Fabeln und Schwänken des Hans Sachs". Dorothea Klein (Hg.): Vom Verstehen deutscher Texte des Mittelalters aus der europäischen Kultur: Hommage à Elisabeth Schmid. Würzburg: Königshausen \& Neumann 2011, 465-474.

Duden. Bd. 7: Etymologie. Herkunftswörterbuch der deutschen Sprache. Mannheim / Wien / Zürich: Bibliographisches Institut 1963.

Frenz, Dietmar: „Giovanni Boccaccio“. Kindlers Literatur Lexikon. 3., völlig neu bearbeitete Auflage. Hrsg. von Heinz Ludwig Arnold. Stuttgart: J. B. Metzler'sche Verlagsbuchhandlung / Carl Ernst Poeschel Verlag 2009, 685-695.

Grimm, Jacob u. Wilhelm Grimm: Deutsches Wörterbuch. Bde. 5, 8. Leipzig: Verlag von S. Hirzel, 1873, 1893. (DWB)

Hahn, Reinhard: „Hans Sachs“. Stephan Füssel (Hg.): Deutsche Dichter der Frühen Neuzeit 14501600. Berlin: Erich Schmidt Verlag 1993, 406-427.

Hartmann, Julius: Das Verhältnis von Hans Sachs zur sogenannten Steinhöwelschen Decameronübersetzung. Berlin: Meyer \& Müller 1911.

Hedwig, Klaus. „Zorn. I.: Philosophisch-theologisch“. Lexikon des Mittelalters. Bd. IX. München: Deutscher Taschenbuch Verlag 2002, 674-675.

Isenring, Johannes: Der Einfluß des Decameron auf die Spruchgedichte des Hans Sachs. Genf: Imprimerie du Courrier 1962.

Jakobs, Béatrice: Rhetorik des Lachens und Diätetik in Boccaccios Decameron. Berlin: Duncker \& Humboldt 2006.

Jauß, Hans-Robert: „Alterität und Modernität der mittelalterlichen Literatur“. Ders.: Alterität und Modernität der mittelalterlichen Literatur. Gesammelte Aufsätze 1956-1976. München: Fink 1977, 9-46.

Kocher, Ursula: Boccacio und die deutsche Novellistik. Formen der Transposition italienischer ,novelle' im 15. und 16. Jahrhundert. Amsterdam / New York: Rodopi 2005.

„'Interpres rerum tuarum': Boccaccio und Petrarca, eine ungleiche Freundschaft“. Karl A. E. Enenkel u. Jan Papy (Hgg.): Petrarch and His Readers in the Renaissance. Leiden: Brill 2006, 53-71.

Könneker, Barbara: „Ehemoral in Fastnachtspielen“. Horst Brunner, Gerhard Hirschmann u. Fritz Schnelbögl (Hgg.): Hans Sachs und Nürnberg..., 219-244.

„Sachs, Hans“. Literatur Lexikon. Autoren und Werke deutscher Sprache. Hrsg. von Walter Killy. Gütersloh / München: Bertelsmann Lexikon Verlag 1991, 99-102.

Kugler, Hartmut: „Selbstdarstellung und Gemeinschaftsleben“. Werner Röcke u. Marina Münkler (Hgg.): Die Literatur im Übergang vom Mittelalter zur Neuzeit ..., 409-419. 
Meid, Volker: Metzler Chronik Literatur. Werke deutschsprachiger Autoren. Stuttgart / Weimar: Verlag J. B. Metzler 2006.

Meuer, Marlene: „Petrarcas Begründung der humanistischen Moralphilosophie: Rezeption und Relativierung der stoischen Tradition“. Barbara Neymeyr, Jochen Schmidt u. Bernhard Zimmermann (Hgg.): Stoizismus in der europäischen Philosophie, Literatur und Politik. Eine Kulturgeschichte von der Antike bis zur Moderne. Bd. 1. Berlin / New York: De Gruyter 2008, 425-452.

Münkler, Marina: „Volkssprachlicher Früh- und Hochhumanismus“. Werner Röcke u. Marina Münkler (Hgg.): Die Literatur im Übergang vom Mittelalter zur Neuzeit ..., 77-96.

Röcke, Werner u. Marina Münkler (Hgg.): Die Literatur im Übergang vom Mittelalter zur Neuzeit. München / Wien: Carl Hanser Verlag 2004.

Röcke, Werner: „Die getäuschten Blinden. Gelächter und Gewalt gegen Randgruppen in der Literatur des Mittelalters“. Werner Röcke u. Hans Rudolf Velten (Hgg.): Lachgemeinschaften. Kulturelle Inszenierungen und soziale Wirkung von Gelächter im Mittelalter und in der Frühen Neuzeit. Berlin / New York: De Gruyter 2005, 61-82.

Suchomski, Joachim: „Delectatio“ und „utilitas“. Ein Beitrag zum Verständnis mittelalterlicher komischer Literatur. Bern / München: Francke 1975.

Theisen, Joachim: Arigos Decameron. Übersetzungsstrategie und poetologisches Konzept. Tübingen / Basel: Francke Verlag 1996. 